\title{
Towards the Development of Smart Sensors to Prevent the Failure of Concrete Infrastructure Components
}

\author{
Daniel J. Thomas · John Kinuthia
}

Submitted: 25 June 2017/Published online: 21 July 2017

(C) ASM International 2017

As of 2017, there are approximately 235,000 bridges made of conventional reinforced concrete in the USA. Currently, 30 percent of these bridges are structurally deficient or functionally obsolete. Contemporarily, there are the unsung efforts of tens of thousands of engineering professionals who continue to assess many of our nations bridges, railway and highway infrastructure, which is an ongoing and unending task. Despite these efforts, much of this infrastructure, which was constructed during the depression era years, is now naturally coming toward the end of its life and needs to be renewed.

Concrete is the most important material that has played a vital part in forging this nation and progressing it from despair to prosperity. Although a durable and dependable material, concrete has intrinsic limitations which can lead to subsequent failure. Complexities such as the conditions during the pour, the utilization of irregular aggregates, environmental factors, the formation of high internal stress concentrations and bond failure can all have disastrous consequences many years down the line. But there is often no way of detecting a failure event before it happens. Critical issues that can result in failure are shown in Figure 1, these include:

1. Corrosion of steel reinforcement when exposed to rock salt used for roadway deicing. Here the deterioration

D. J. Thomas $(\bowtie)$

School of Engineering and Applied Science, Yale University, New Haven, CT 06520, USA

e-mail: daniel.thomas@engineer.com

\section{J. Kinuthia}

Faculty of Computing, Engineering and Science, University of South Wales, Llantwit Road, Pontypridd, UK

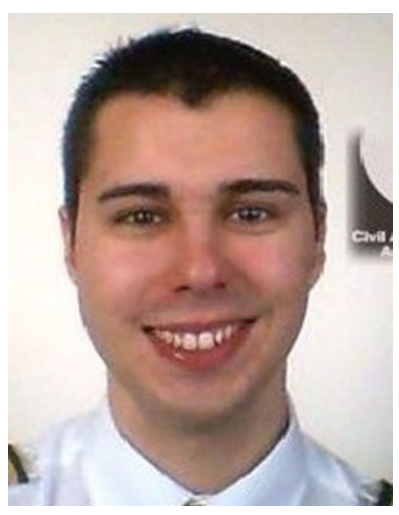

Daniel J. Thomas

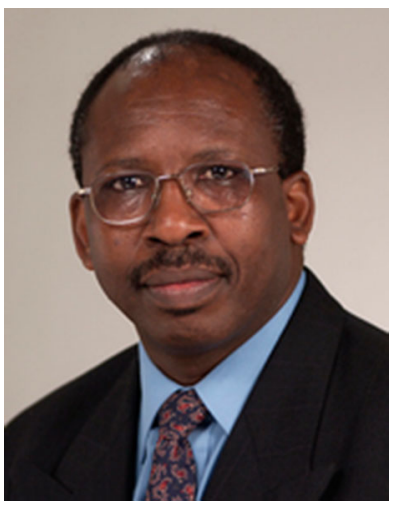

John Kinuthia

occurs because the by-product of this electrochemical process is iron-oxide, which increases the volume of the uncorroded steel. The resulting pressure created inside the structure which causes cracking and subsequent deterioration to the structure. Further to this, there are also effects of carbonation, where carbon 
dioxide and moisture in the air enter the concrete and reduce the $\mathrm{pH}$ of the concrete also resulting in corrosion of the steel reinforcement.

2. Sulfate attack occurs when the concrete is exposed to water that contains a high concentration of dissolved sulfates. This is prevalent in Western states, near industrial areas and marine regions. Sulfate-containing water enters the surface of the concrete, crystallizes and expands. This subsequently disrupts the hardened concrete and causes chemical attack, where the sulfate salts react causing it to dissolve, soften and erode the cement.

3. Finish-related delamination occurs either water or air gets accumulated below the surface of the concrete. This accumulation of water raises the ratio of water-tocement, which significantly decreases the concrete strength.

4. Freeze-thaw deterioration occurs when concrete is saturated with moisture while exposed to freezing temperatures. This occurs particularly in the Pacific North West and North East coast. The freezing water within the concrete creates hydraulic pressures within the structure, which causes the formation of microfractures. The surface region of the concrete subsequently crumbles, exposing the aggregate. The knock-on effect of this is that the whole structure will subsequently fail.

5. Alkali-silica reactions can also occur when certain silica-containing aggregates react to form an expansive gel that causes the concrete to crack. The cracks typically form in a widespread pattern, which causes localized crumbling in the structure. This often results in catastrophic failure.

These factors are critical toward influencing the integrity of concrete structures. As the renewal of the old concrete infrastructure takes place over the next twenty-years. These projects will become the biggest combined construction project ever to be undertaken in the history of human civilization. A question that comes to mind is over the next seventy to eighty years, where are the army of engineers, technicians and skilled work men/women who will inspect, maintain and service this infrastructure going to come from? Because, as the next generation of heavy infrastructure projects starts, it is good to learn from the past. And ensure that we can build condition monitoring into concrete components to prevent them from failing. This way they will last for many future generations without relying on unsustainable demands on labor.

Currently, the construction industry does not have a robust identifiable approach or means of using large area monitoring for the structural integrity of concrete systems. This creates a gap in the monitoring of real-time loading stresses and strains in the structure, all of which would benefit from future integration of embedded sensors. There is also no fundamental technology, which has been developed to perform the task of continuous structural monitoring. This needs to be in the form of large area strain sensors, which can be used to monitor microstrain in the concrete structure. As a result, large area customisable
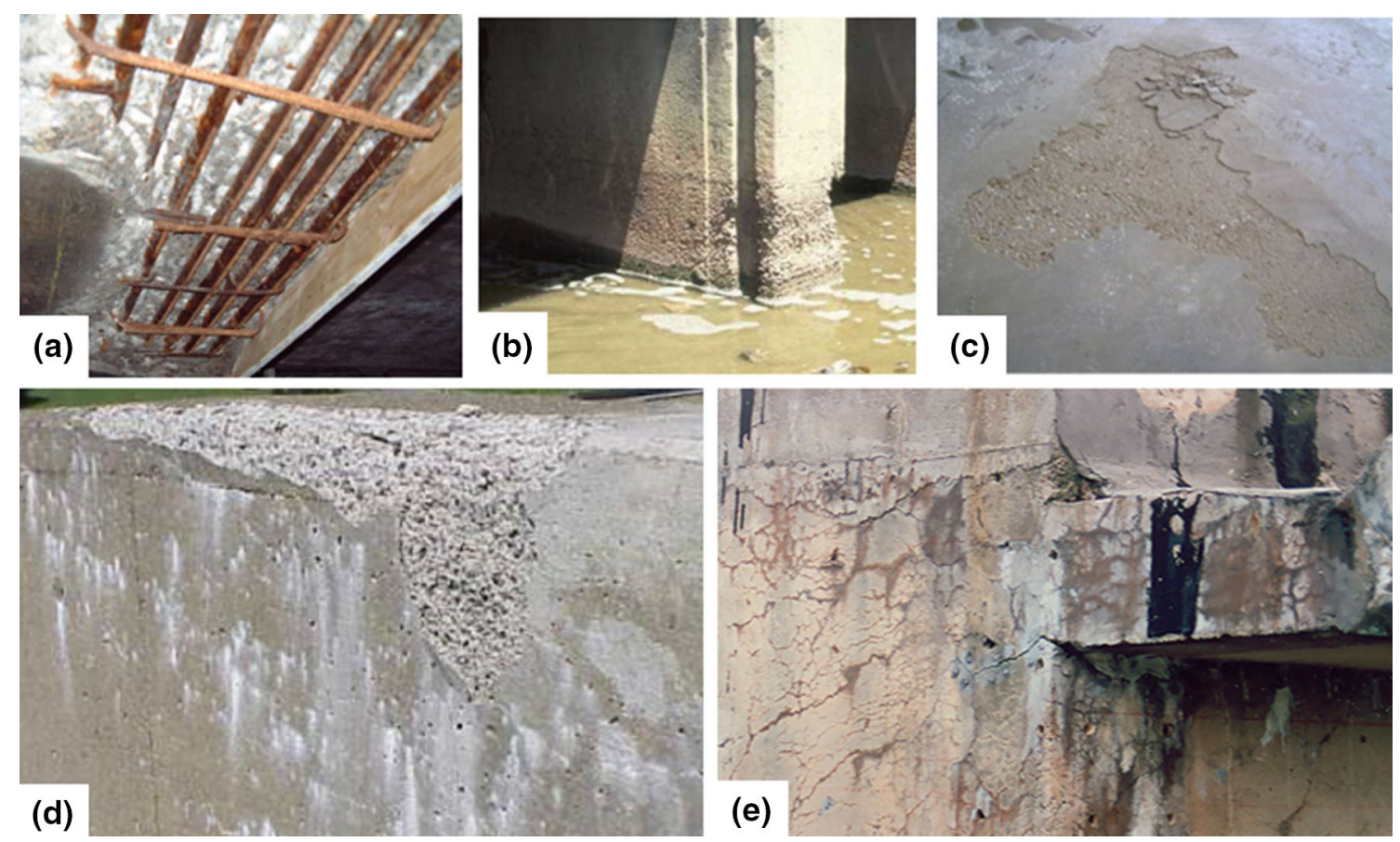

Fig. 1 Showing the main failure mechanisms of concrete structures. (a) Corrosion of steel reinforcement. (b) Sulfate attack. (c) Finish-related delamination. (d) Freeze-thaw deterioration and (e) Alkali-silica reaction 
conformable sensors can then be integrated during (1) the construction phase or (2) during repair of the structure.

This method offers novel means toward real-time monitoring. The ability to monitor the integrity of concrete structures is a vital component toward ensuring structural integrity. The long-term savings by obviating post-construction, in which intergration to structures in postinstallations are attractive in sustainably maintaining the integrity of new infrastructure components.

The biggest issue is in developing durable methods of autonomously monitoring the structural integrity of concrete civil structures in situ throughout its life. As a result, embedded sensors have a direct potential toward monitoring the whole life of both precast and onsite cast concrete systems. This offers a new solution to an innovative market, which can result in the rapid deployment of technology.

Future avenues of sensor system integration can include the detection and monitoring of; temperature, moisture, humidity, chemical monitoring and $\mathrm{pH}$. The existing practice in construction has no installation of electronic monitoring devices. These in situ monitoring processes offer a next generation approach toward advanced forms of manufacturing in the fabrication of smart failure proof concrete.

In order to develop autonomous methods of monitoring concrete structures, then core elements are required, in the form of:

- Sensor networks and communications: These need to be large area, with sensor configuration and positioning, together with data compression algorithms to provide reliability.
- Sensor data acquisition: Scalable strain-based sensor for localization of damage and also multi-site damage localization.

- Power Management: There need to be a trade-off between sensing and communications, which will need to be instigated through the use of smart energy-aware activation circuits for interfacing wireless sensing systems to reduce power consumption.

- Energy Harvesting: Novel power harvesting hardware is needed throughout the life of the application.

These methods to monitor the performance of concrete structures can also be used to validate the performance of novel greener concrete formulations. These can be used as a novel means toward ensuring structural durability. In situ monitoring of concrete also provides the basis for monitoring the integrity of earthquake proof structures for internal structure failure.

Future embedded sensor technology directly addresses durability opportunities of lifetime monitoring. The ability to monitor creep, deformation, stresses and strains in materials, elements and entire structures will increase. The ability to monitor concrete structures over the whole life is a radical and holistic approach toward failure prevention. It also has significant cost and time savings, which will increasingly become attractive.

The proposed approach of concrete monitoring does add complexity to the controlled manufacturing conditions. But it will be possible to address futuristic whole life monitoring of new structures to prevent their failure. 